\title{
Docking Study of Biflavonoids, Allosteric Inhibitors of Protein Tyrosine Phosphatase 1B
}

\author{
Jee-Young Lee, Ki-Woong Jung, Eun-Rhan Woo, and Yangmee Kim* \\ Deparment of Bioscience and Biotechnology, Biolfolectlar Informatics Center, Konkuk Lniversity, Seoul 143-701, Korea \\ ${ }^{*}$ E-mail: mkimakikonkk.ac.kr \\ College of Pharmacy, Chosu Lninersitw, Guangin 501-759, Korea \\ Received June 13,2008
}

\begin{abstract}
Protein ty rosine phosphatase (PTP) IB is the superfamily of PTPs and a negative regulator of multiple receptor ty rosine kinases (RTKs). Inlibition of protein tyrosine phosphatase IB (PTPIB) has been proposed as a strategy for the treatment of type 2 diabetes and obesity. Recently. it has been reported that amentoflavone. a biflavonoid extracted from Selaginella tamariscina inhibited PTPIB. In the present study, docking model between amentoflavone and PTPIB was determined using automated docking study. Based on this docking model and the interactions between the known inhibitors and PTPIB, we determined multiple pharmacophore maps which consisted of five features. two hydrogen bonding acceptors. two hydrogen bonding donors. and one lipophilic. Using receptor-oriented pharmacophore-based in silico screening. we searched the biflavonoid database including 40 naturally occurring biflavonoids. From these results. it can be proposed that two biflavonoids. sumaflavone and tetrahydroamentoflavone can be potent allosteric inhibitors. and the linkage at $5^{\prime} .8$ "-position of two flavones and a hydroxyl group at 4'-position are the critical factors for their allosteric inhibition. This study will be helpful to understand the mechanism of allosteric inhibition of PTPIB by biflavonoids and give insights to develop potent inhibitors of PTPIB.
\end{abstract}

Key Words : PTP1B. Biflavonoid, Docking study, In silico screening, Allosteric site

\section{Introduction}

Protein tyrosine phosphatase (PTP) 1B is the superfamily of PTPs and a negative regulator of multiple receptor tyrosine kinases (RTKs). ${ }^{\text {l: }}$ Also PTPIB is involved in the downregulation of insulin and leptin signaling. Thus. inhibitors of PTP 1B have potential as therapeutics for treating Type 2 diabetes and obesity. ${ }^{23}$ Recently. it has been reported that PTPIB inhibition may lead to increased oncogenic signaling. ${ }^{4-6}$ Therefore. PTP IB is increasingly drawing attention as an attractive target for anticancer as well as diabetes and obesity:

In the strategy of design of PTP1B inhibitors. selectivity between PTPs is very important factor. The catalytic site of PTPs is highly conserved and has the intractability to small molecule drug discovery thus the investigation of other mechanisms of inhibition is prompted. At 2004, a novel allosteric site in PTP1B was discovered by Hansen group. ${ }^{7}$ Since this site is not well conserved among phosphatases. it afforded an opportunity to outwit the problems associated with inhibition of catalytic site. Allosteric inhibition is a promising strategy for targeting PTPIB and constitutes a mechanism that may be applicable to other tyrosine phosphatases. $^{\text {? }}$

According to the recent report. amentoflavone. a naturally occurring biflavonoids derived from Selaginella tamariscina. inhibited activity of PTP1B by allosteric inhibition. ${ }^{\S} \mathrm{Bi}-$ flavonoids are the dimer of flavonoids (homo or hetero) connected with a C-C or C-O-C bond ${ }^{5}$ Many different combinations of flavonoids are possible and may result in various chemical structures. For example. flavanone-flavone. flavone-flavone. and flavanone-flavonol are the most conmon biflavonoids with connecting linkages at diverse positions. In natural biflavonoids. hydroxy $1 /$ methoxy groups are substituted at different positions. Even though numerous biflavonoids with various combinations of flavonoids can be possible. plants that contain biflavonoids as major constituents are not widely distributed. ${ }^{9.10}$ More than 100 biflavonoids have been identified from plants and a variety of biological activities of biflavonoids have been published. including anti-inflammatory. antimicrobial. antioxidant activities. ${ }^{11-1.3}$ A symmetric biflavone, amentoflavone is a homo-dimer of apigenins and has several known pharmacological activities such as anti-inflammatory. antioxidative. and anticancer effects. $^{14.15}$

Here, automated docking study for PTP1B was performed and a docking model between PTPIB and amentoflavone at the allosteric site of PTP1B was proposed. Furthermore. we determined a pharmacophore map by receptor-oriented pharmacophore-based in silico screening using structurebased focusing (SBF) module of Cerius $2^{(k-1)-2 !}$ and screened potent biflavonoid inhibitors.

\section{Methods}

Docking study. Docking study was performed between amentoflavone and PTPIB in order to find specific binding model using AutoDock ${ }^{21.22}$ based on $\mathrm{x}$-ray structure of PTP1B (IT49.pdb and 1T4J.pdb). The Lamarckian Genetic Algorithm (LGA) of the AutoDock 3.05 was used for 
docking experiments. Distance-dependent function of the dielectric constant was used for the calculation of the energetic maps. MD simulations were performed for the final docking structures in the canonical ensemble (NVT) at $300 \mathrm{~K}$ using the program InsightII/Discover. All atoms of the system were considered explicitly, and their interactions were computed using the consistent valence force field. A distance cutoff of $10 \AA$ was used for van der Waals interactions and electrostatic interactions. The time step in the MD simulations was $l \mathrm{fs}$ and $\mathrm{MD}$ sumulation was performed for $2 \mathrm{~ns}$. Coordinates were saved every $1 \mathrm{ps}$.

Receptor-oriented Pharmacophore-based in silico Screening. The interaction model used for this process is a list of features, such as hydrogen bonds and lipophilic interactions. These features include hydrogen bond donors (HBDs), hydrogen bond acceptors (HBAs) and lipophilicity (Lipo).

We defined the active site of PTPIB using the center and the radius of the docked inhibitor and amentoflavone. An interaction model was generated within $10 \AA$ of the center of

Table 1. Chemical structures of representative biflavonoids in our database

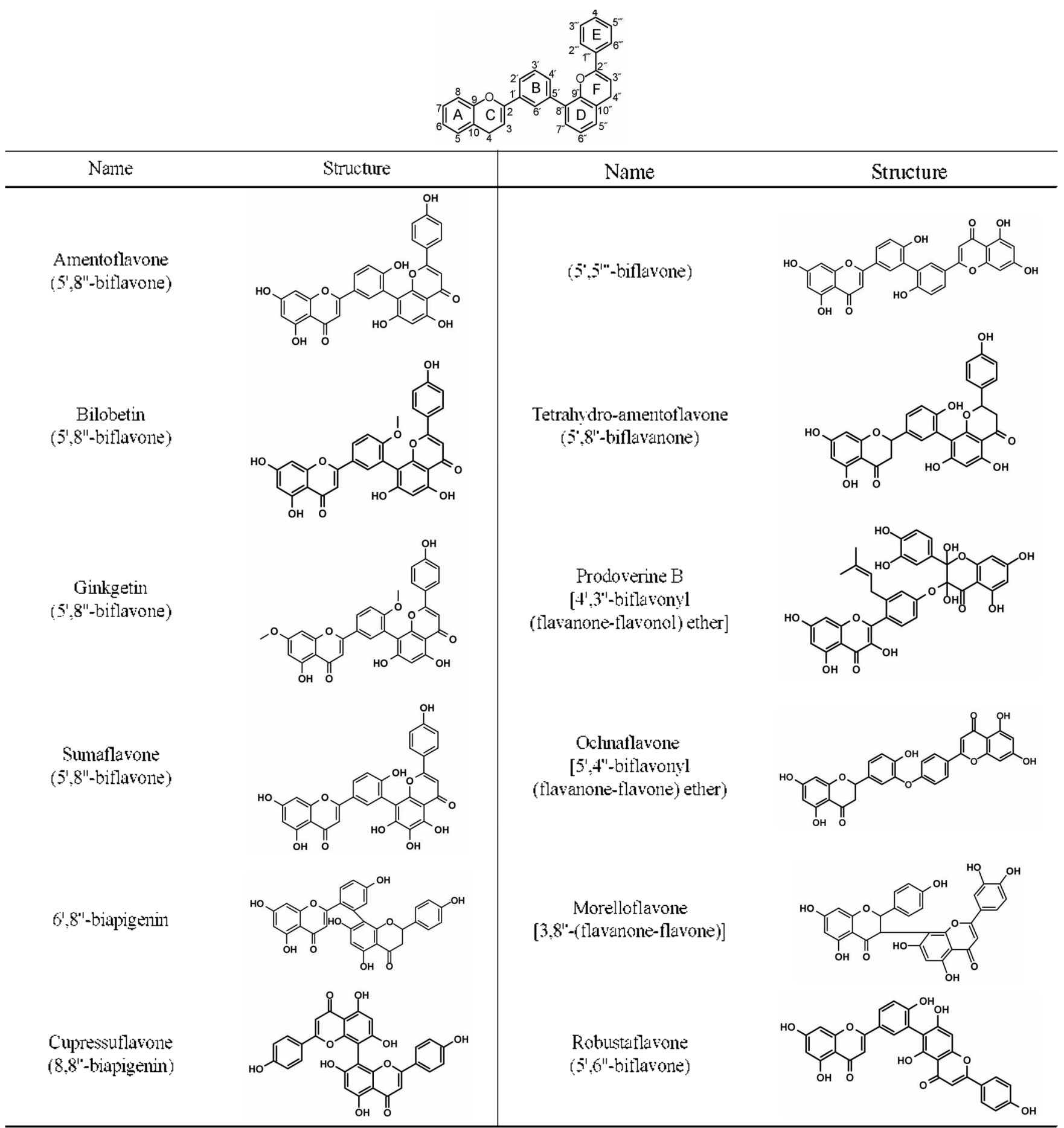


the active site. Multiple pharnacophore maps were determined with five features for each map and the exclusion volume. The exclusion volume was built from the heavy atoms within $10 \AA$ of the center of the active site. Then. pharmacophore map which reproduced the docking model of PTPIB and amentoflavone was selected. We built a database of 40 biflavonoids with different combinations of flavonoids as listed in Table 1. such as flavanone-flavone. flavone-flavone. flavanone-flavonol. ${ }^{-3.41}$ This database was browsed using selected pharmacophore map. The scoring functions (LigScore2, PLP1, and PLP2) were calculated for hits to establish a relationship between this pharmacophore and the set of inhibitors using Cerius $2 .+2,43$

\section{Results and Discussion}

Analysis of x-ray structure of PTP1B and allosteric inhibitor. The catalytic site of PTPIB is centered at Cys 215 residue and included WPD loop. which closure is essential for the catalytic mechanism of PTPIB. Allosteric site of PTPIB is located $\sim 20 \AA$ away from Cys 215 at the catalytic active site. ${ }^{7}$ The interactions between PTPIB and allosteric inhibitors have been identified from the X-ray crystal structures and we utilized this information to analyze the docking model of PTPIB and amentoflavone. Two active sites of PTPIB. catalytic site and allosteric site, are represented in Figure 1. X-ray structures of PTP IB showed that allosteric inhibitors (inhibitor 2 and 3) formed several important hydrogen bonds with PTP IB. ${ }^{7}$ Hydrogen bonding interactions are observed with the side chain of Asn 193 and the carboxyl oxygen of Glu276. In addition. one watermediated hydrogen bonding between hydroxyl group of inhibitor and the main chain carbonyl of Phel96 is formed. Two allosteric inhibitors particularly wrap around Phe280 and these hydrophobic interactions, combined with the hydrogen bonding with Glu276, probably increase their potency. ${ }^{7}$ In the case of inhibitor 1 . this nolecule was not wrapping the hydrophobic residue Phe 280 and did not formed a hydrogen bond with Glu276. This is a reason for the low inhibitory activity of inhibitor 1 on PTP1B. The binding model between inhibitor 2 and PTP1B is shown in
Figure 1 .

Docking study between PTP1B and amentoflavone. Docking model of amentoflavone and PTPIB was similar to $\mathrm{x}$-ray structures of PTPIB complex with known inhibitors. and several hydrogen bonds were also found in our docking model. 4-carboxyl group of amentoflavone and side chain of Asn 193 formed a hydrogen bonding. and 4'-hy drosyl group and side chain carboxyl oxygen of Glu276 also participated in hydrogen bonding interaction. It is already known that the hydrogen bonding between inhibitor and Glu276 has significant effect to increase the inhibitory activity. 7-hydroxyl group of amentoflavone is participated in water mediated hydrogen bond interaction. In addition. hydroxyl group at 7"'-position of amentoflavone formed a hydrogen bonding with backbone oxygen of Phe 280 and this hydrogen bonding was not found in the $\mathrm{x}$-ray structures of PTPIB complex with three inhibitors. Thus. this is a unique feature of biflavonoids inhibitors of PTPIB. Amentoflavone also wrapped around Phe 280 and formed hydrophobic interaction with Phel96 and Leu 192. The interaction model between amentoflavone and PTPIB is shown in Figure 2A

Receptor-oriented pharamcophore-based in silico screening. Based on docking structure, we generated a receptororiented interaction model for the active site of PTPIB and five phamrcophore maps were determined based on the hydrogen bonding interactions and lydrophobic interaction between PTPIB and known allosteric inhibitors. Five maps consisted of five features, two HBA including a water mediated hydrogen bonding with Phe 196 and a hydrogen bonding with Asn L93. two HBD including a hydrogen bonding with Glu276 and that with backbone carbonyl of Phe280. and one Lipo including hydrophobic interaction with Leul92 and Phe280. Among five features in pharmacophore map, three features including two HBA and one HBD were satisfied with the interactions between PTPIB and known inhibitors. To define a proper Lipo site. we determined five multiple maps which have a Lipo site at different coordinate in allosteric site. Among these five maps. only Map 3 reproduced the binding model of amentoflavone and PTP $1 \mathrm{~B}$ properly and Map 3 is shown in Figure 2B.

Structural data of 40 known biflavonoids were collected

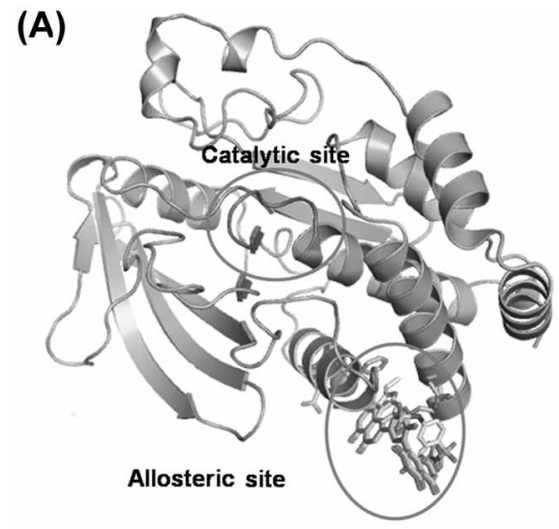

(B)

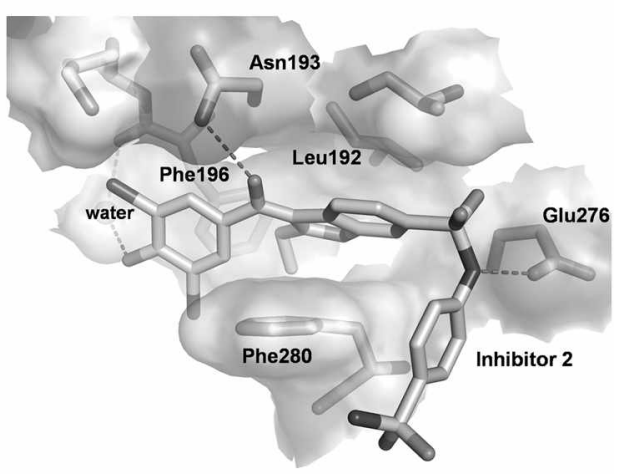

Figure 1. (A) Crystal structure showing the catalytic site and allosteric site of PTP1B. (B) Binding structure of PTP1B and inhibitor 2 in allosteric site? 
(A)

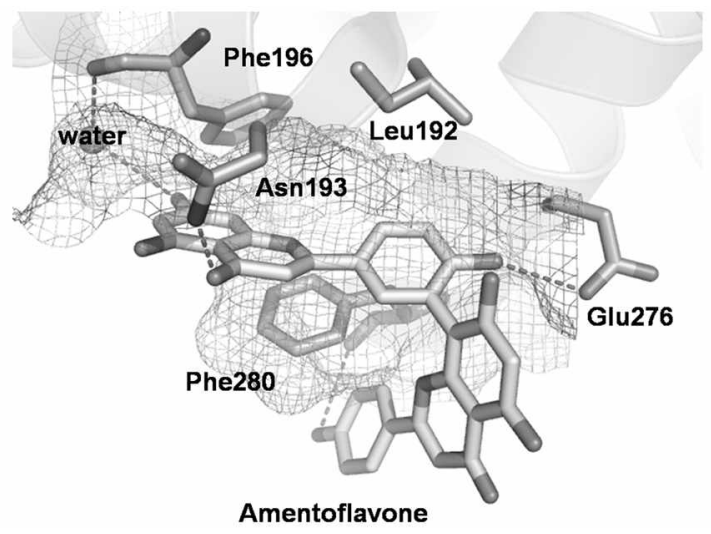

(B)

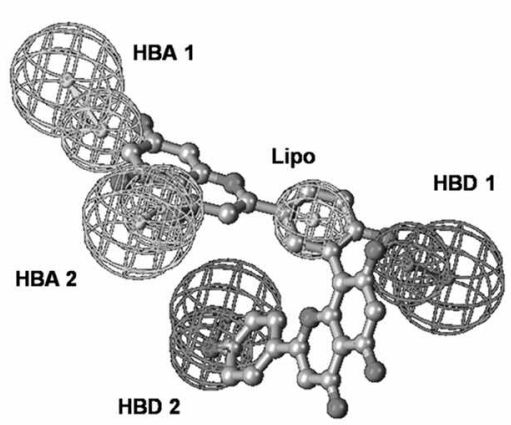

Figure 2. (A) Docking model of PTPIB and amentoflavone. Side chain of Phe196, Leu192, and Phe280 participated in hydrophobic interactions with amentoflavone. (B) Optimal pharmacophore map, Map 3.

(A)

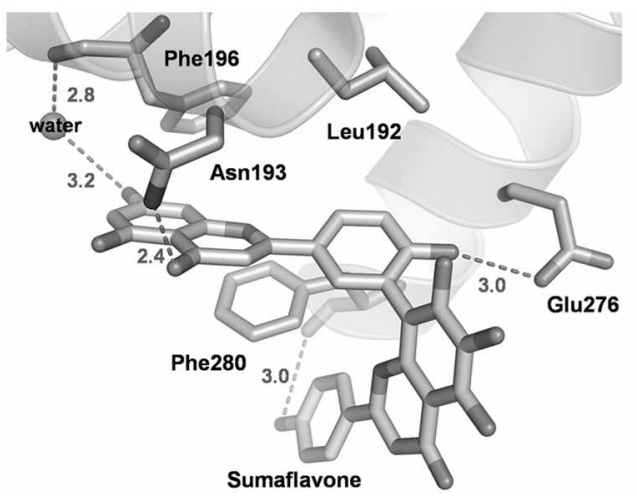

(B)

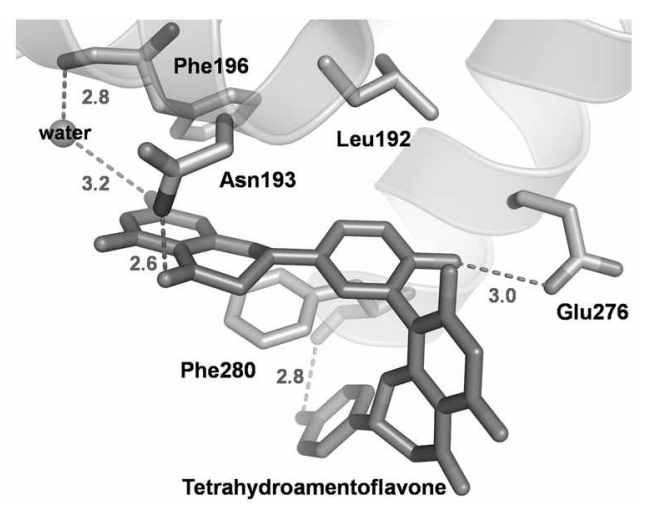

Figure 3. Hit models of biflavonoids and PTP1B. (A) Sumaflavone (B) Tetrahy dro-amentoflavone

from several articles and biflavonoid database. ${ }^{\hat{.} \hat{3} \cdot 41}$ These are naturally occurring biflavonoids and they clustered by combinations of flavanone-flavone, flavone-flavone. and flavanoneflavonol with various linkages. Chenical structures of representative 12 biflavonoids are depicted in Table 1 . We built bioflavonoid database and then searched it with Map 3. Among 40 biflavonoids. only two. sumaflavone and tetrahydroamentoflavone. matched well with the phamacophore map. Sumaflavone is an asymmetric biflavones isolated from plants such as Selaginella tamariscina. ${ }^{23}$ Tetrahydroamentoflavone (THF) is one of the major flavonoids extracted from Semecarpus ancacardium Linn. (Anacardiaceae) ${ }^{34}$ These two biflavonoids have potent anticancer activity. ${ }^{13.24}$ Hit models of two biflavonoids satisfied each features of phanracophore maps with proper configurations. Interactions between PTPIB and these two biflavonoids and the distances between the atoms participated in hydrogen bonding interactions are shown in Figure 3.

Amentoflavone. sumaflavone, and THF are biflavone linked at $5^{\prime} \cdot 8^{\prime \prime}$ position. Amentoflavone is $5^{\prime} .8^{\prime \prime}$-biapigenin and sumaflavone is constructed of one apigenin and $4^{\prime}, 5.6 .7-$ tetrahydroxy flavones linked at $5^{\prime}, 8^{\prime \prime}$ position. The chemical name of THF is $5^{\prime} 8^{\prime \prime}$-binaringenin. Other biflavonoids which do not have 5' 8 " linkage could not bind suitably at the allosteric site of PTP1B because of the steric hindrance and could not wrap Phe 280 properly. Even though ginkgetin is a biflasonoid with $5^{\prime}, 8^{\prime \prime}$ linkage. methy lation of $4^{\prime}-\mathrm{OH}$ disrupt- ed the hydrogen bond with Glu276 and the water mediated hydrogen bond is missed by methylation of $5-\mathrm{OH}$. Bilobetin also missed the hydrogen bonding interaction with Glu276 because of methylation of 4'-position.

From these results. linkage type of biflavonoids is the critical factor for the inhibitor to wrap Phe280 properly. A hydroxyl group on 4'-position of biflavonoids provides the stable hydrogen bonding with Glu276 which is the essential feature for allosteric inhibition. Additionally $4^{\prime \prime \prime}-\mathrm{OH}$ of these biflavonoids form an extra hydrogen bonding with the backbone carbonyl of Phe 280 and increase the inhibitory activity of them.

Evaluation of docking study between PTP1B and biflavonoids. To verify docking study. we calculated scoring functions: LigScore2. PLP1, and PLP2. The LigScore2 is a scoring function that possesses high predictive accuracy of affinity of ligand-receptor binding as well as $p K_{i}$ values. ${ }^{42}$ The PLP (Piecewise Linear Potential) is an empirical scoring function with two types. PLPI and PLP2. In the PLPI function. each non-hyddrogen ligand or non-hydrogen receptor atom is assigned as PLP atom types. All hydrogen atoms are excluded from the PLP function. In PLP2. PLP atom type remains the same as in PLPl, but a PLP atomic radius is assigned to each atom except for lydrogen. thus atomic interactions represent the hydrogen bonding. repulsion. and dispersion. In both PLP functions. the higher PLP score indicates the stronger binding affinity with the receptor. ${ }^{43}$ 
Table 2. The scoring function LigScore2 and PLP of known inhibitors and biflavonoids

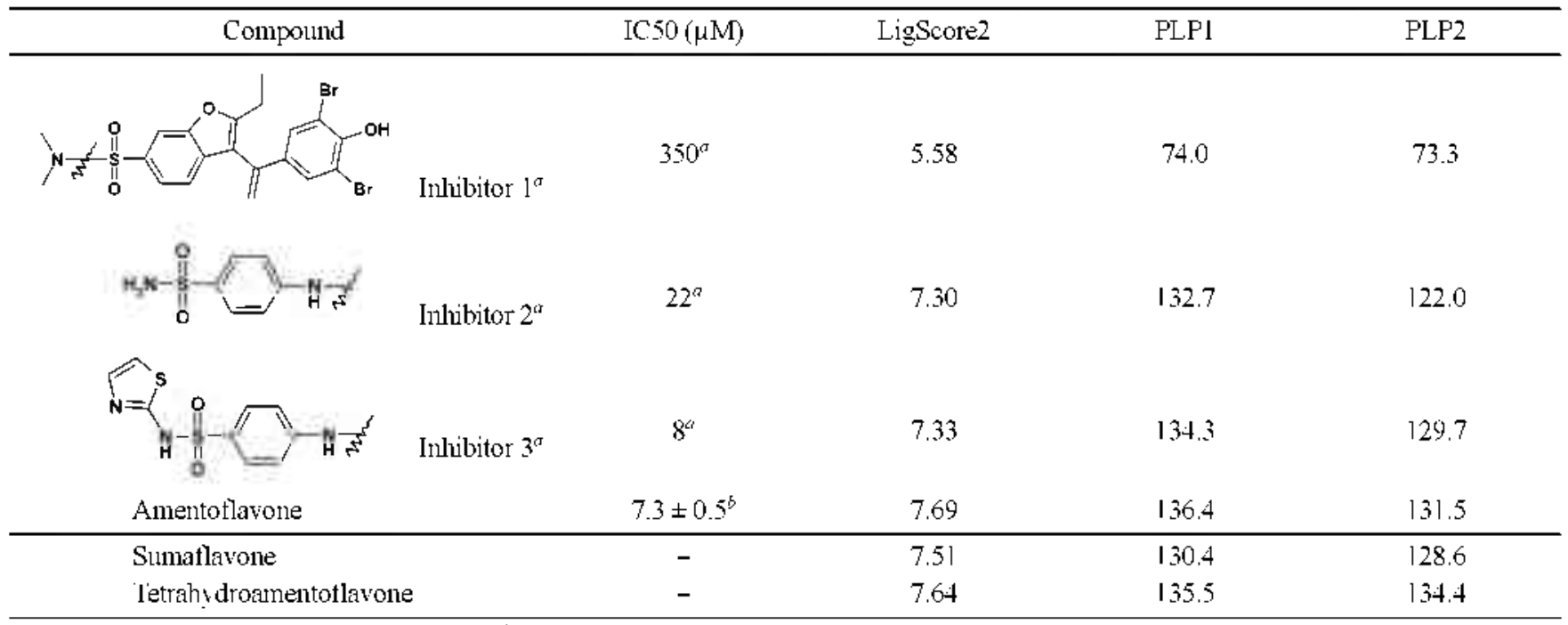

"Christian. W. et al. Nat. Struc. Mol. Biol. 2004. 'Na. M. K. et al. Biol. Phan Bull. 2007.

Experimental $\mathrm{IC}_{5}$ value of allosteric inhibitors and amentoflavone reported previously, 7,8 and the calculated scoring functions (LigScore2. PLPI. and PLP2) of known inhibitors and hit biflavonoids are conpared in Table 2.

All three scoring functions of anentoflavone and inhibitor 3 which have the best $\mathrm{IC}$ (5) values are highly ranked than the rest two inhibitors. The least active inhibitor (inhibitor l) has the lowest scores as listed in Table 2. Scoring functions. especially PLP 2. proved that autonated docking process for amentoflavone and PTPIB was well performed and the docking model represented well the critical features for inhibitors of PTPIB. It can be proposed that hit biflavonoids. sumaflavone and THF, in this study can be potent allosteric inhibitors of PTPIB.

\section{Conclusion}

In this study. we determined the docking model between amentoflavone and PTP1B using automated docking study and we confirmed that amentoflavone was well docked in the allosteric site of PTP1B. Based on docking model. we detemined multiple phanmacophore maps using by receptororiented pharmacophore-based in silico screening and determined optimal map which consisted of five features: two HBA two HBD. and one Lipo. We searched biflavonoid database including 40 naturally occurring biflavonoids with this map. Docking study and receptor-oriented phammacophore based in silico screening. it was proposed that two biflavonoids. sumaflavone and tetrahydrowamentoflavone can be potent allosteric inhibitors of PTP1B. where two flavones are linked at 5'.8'-position and have a hydroxyl group at 4'-position. These results designated that certain biflavonoids can affect cancer disease. suggesting therapeutic potential against cancer. Further study, we will investigate further anticancer activity of these biflavonoids for several anticancer targeted proteins. protein tyrosine phosphatases such as PTPIB and PRL-3. This study may provide a strategy for the development of novel PTPIB allosteric inhibitors.

Acknowledgments. This work was supported by the Research Program for New Drug Target Discovery (M10601000153-07N0100-15310) grant from the Ministry of Science \& Technology, South Korea and by $\mathrm{Bio} /$ Molecular Informatics Center of Konkuk University (KRF2006-005-J03402). Ki-Woong Jung is supported, in part, by the second BK2I (MOE).

\section{References}

1. Ostmant. A.: Bohmer. F. Trends Cell. Biol. 2001. 11. 258.

2. Li. S.: Depetris. R. S.: Barford. D.: Chernoff. J.: Hubbard. S. R. Shicture 2005. 13. 1643

3. Dixit, M.: Iripathi, B. K.; Tamrakar. A. K: Srivastava. A. K: Kumar. B:- Goel, A. Bioorg. 1ed. Chem $2001,15,727$.

4. Y. T.; Lindner. D. Cwr: Onco. Report 2008. 10. 114.

5. Dube. N.: Cheng. A.: Tremblay: M. L. PN. $4 S$ 2004. 101. 1834.

6. Lyon. M. A.: Ducruet. A. P.: Wipt. P.: Lazo. T. S. Nat. Rev 2002. 1. 961 .

7. Wiesmann. C.: Barr. K. J: Kung. J.: Zhu, J.: Erlanson. D. A.: Shen, W: Falur. B. J.: Zhong. M.: Taylor. L.: Randal, M.; MeDowell. R. S.: Hansen. S. K. Nat. Stuc. Hol Biol 2004, 11.730.

8. Na. M.: Kim. K. A.: Oh. H.: Kimn. O. Y: Oh. W. K.: Ahn. T. S. Biol. Phom. Bull. 2007. 30.379.

9. Chen. J.: Chang. H. W.: Kim. H. P.: Park. H. Bioong Med Chent Lett. 2006, 16. 2373 .

10. Kim. H. P. Park. H; Son. K. H; Chang. H. W.: Kang, S. S. Arch Pham Res. 2008, 31. 265.

11. Lin. Y.: Flatin. M. T.: Cassidy. C. S.: Mar. A.: Chen. F. Bioorg Med Chem. Letl. 2001. H. $2 \mathrm{I}$ (1).

12. Blazso. G.: Gabor. M.: Rohdewald. P. Phamazie. 1997. 52. 380.

13. Ursini, F.: Rapuzzi. I.: Toniolo, R:- Tubaro, F.: Bontempelli, G. Methods Enzwnol. 2001, 335, 338 .

14. Woo, E. R.: Lee, J. Y:: Chom. I. J.; Kim. S. G.; Kang, K. W. Pharnaco. Res. 2005.51 .539

15. Pant. X.: Tan. N.: Zeng. G.: Zhang. Y.: Tia. R. Bioorg. Med Chent. 2005. 13. 5819.

16. Paul. D. K.: Rob. B.: Scott, K: Marvin. W: Venkatachalam. C. M. J. of Conp. Chem. 2001, 22,993. 
17. Hotfren. A. M.: Murray. C. M.: Hottmann. R. D. Cur: Pharm. Des. 2001. 7.547.

18. Luke. S. F.: Osman. F. G. J. Braz. Chem. Soc. 2002. 13. 777.

19. Pickett. S. D.; Mason. J. S.; McLay. I. M. J. Chem. Inf. Comput. Sci. 1996, 36. 1214.

20. Lee. J. Y: Baek. S.: Kim, Y. Bull. Nonem them Soc. 2007, 28. 379

21. Morris. G. M.: Goodsell. D. S.: Halliday. R. S.: Huey. R.: Hart. W. E.: Belew. R. K.: Olson. A. J. J. Conputational Chemisty 1998. 19.1639

22. Lee, J. Y.: Lee. S. A.: Kim. Y. Bull Korean (Them. Soc. 2007, 28. 941.

23. Yang. S. F.: Chu, S. C.: Liu. S. J:: Chen. Y. C.: Chang. Y. Z: Hsieh. Y. S. J. Ethopharmaco 2007. 110.483.

24. Gil. R. R.: Lin. L.: Cordell. G. A.: Kumar. M. R.: Ramesh. M.: Reddy: B. M.: Mohan. G. K.: Rao, A. V. N. A. Plytochem 1995. 39.405 .

25. Seltam, C. Sanjay, M. J.J. Ethmophamaco 2004, $95,209$.

26. Li. X.: Hoshi. A. S.: Tan. B.: ElSohly. H. N.: Walker. L. A.: Zjawiony. J. K.: Ferreira. D. Tetrahedron 2002. 58.8709.

27. Bekker. R.: Ferreira. D.: Swart. K. J.: Brandt. E. V. Tetrahedront $2000,56,5297$.

28. Hyun, S. K.: Kang. S. S.: Son. K. H.: Chung. H. Y: Choi, J. S. Chem. Pham. Bull. 2005, 53, 1200.

29. Yamaguchi. L. F: Vassao. D. G.: Kato. M. J.: Mascio. P. D. Plotochemt. 2005. 66.2238.

30. Das. B.: Mahender. G. Rao. Y. K.: Prabhakar. A.: Jagadeesh. B. Chem. Pham. Bull. 2005, $53,135$.

31. Kumar. N.; Singh, B; Bhandarim. P: Gupta, A. P; Uniyal. S. K.:
Kaul. V. K. Phtochem. 2005. 66. 2740.

32. Choi. S. K.: Oh. H. M.: Lee. S. K.: Jeong. D. G.: Ryu. S. E.: Sont. K. H.: Han. D. C.: Sung. N. D.: Baek. N. I.: Kown. B. M. Nat. Product. Res. 2006, 20.341.

33. Lee. C. W. Choi. H. J.: Kim. H. S.: Kim. D. H.: Chang. I. S. Moon. H. T; Lee, S. Y.: Oh. W. K; Woo. E. R. Bioorg Med. Chent 2008, 16.732.

34. Slade. D.: Ferreira. D.: Marais. J. P. I. Phyochent 2005. 66. 2177 .

35. Weniger, B.: Vonthron-Senecheau, C.: Kaiser, M; Brun. R: Anton. R. Plntomedicine 2006, 13. 176.

36. Ariyasena, J.: Baek, S. H.; Perry. N. B.; Weavers, R. T. J. Nat Prod 2004. 67. 693.

37. Mayer. R. Plntochem. 2004. 65. 593

38. Itunocenti. M.: Michelozzi. M.: Giaccherini. C.: Ieri. F.: Vincieri. F. F; Mulinacci. N. J. Agric. Food Chem. 2007. 55.6596.

39. Likhitwitavawuid. K.: Kaewamatawong, R.: Ruangrungsi, N. Biochem. Systemat. Ecology 2005. 33. 527

40. Rampendahl. C.: Seeger. T.: Geiger. H.: Zinsmeister. H. D Phwochen. 1996. H. I621.

41. Howell. H.: Malan. E.: Brand. D. T.: Kamara. B. I.: Bezuidenhoudt. B. C. B.; Marais. C. Steenkamp. J. A. Chemisny of Katmal Compounds 2007. 43.533

42. Krammer. A; Kirchhoff. P. D.; Venkatachalam. X. J. C. M: Waldman. M. J. Mol. Graph Model. 2005. 23. 395.

43. Verkhivier. G. M.: Botzida. D.: Gehlhaar. D. K.: Rejto. P. A.: Arthurs. S.: Colson. A. B.: Freer. S. T.: Larsont. V.: Lutyi. B. A.: Marrone, $T ;$ Rose. P. W. Jownal of Computer-tided I Iolecular Design 2000. 14.731. 